\title{
A CASE OF "SILENT BRONCHIECTASIS"
}

\section{Case Presented to M.R.C.P. Course.}

History. On June 22nd, I934, an English church curate, aged 47, was referred for advice for "persistent bronchitis and asthma." For this condition he had been treated for three years, but had got steadily worse, especially during the last six months.

He complained of a cough which was not relieved by medicine, which was worse on lying down, and especially when he got up in the morning, which was practically the only time he brought up any sputum; it was then about two ounces in amount, and greyish and semi-solid in character. At other times the cough was mostly hard and left him exhausted and breathless, and with much tightness in his chest. Recently exercise had brought on the cough, and he found that even short walks on the flat made him breathless.

Sleep was poor, because of cough and frequent night sweats. Appetite was impaired and in the past two years he had lost twenty-seven pounds in weight. Pain was present on most days in the left chest, at the base anteriorly and in the axilla, and was worse with cough. Sputum had not been examined, and he had not been X-rayed.

Past history contained nothing of note; he had been quite fit until the onset of this condition three years previously.

Such a history was not suggestive of bronchitis and asthma, but much more of a chronic phthisis or of a progressive septic bronchiectasis from stenosis of a bronchus by intrinsic foreign body or extrinsic pressure. Possibility of new growth could not be lost sight of, for while the length of history would militate against a diagnosis of bronchial or so-called true pulmonary carcinoma, pressure by an innocent tumour of the lung of even such a new growth as endothelioma must be considered.

Examination. On examination he was found to have normal pulse and temperature. The chest was poorly covered and was barrel-shaped. The apex beat was normal in position; heart's sounds were normal though distant as if from emphysema. Movement and note were impaired over the bases but there was no definite dullness in any area, and, while there was a generalized emphysema and scattered rhonchi were present, both on inspiration and expiration, no crepitations were heard.

Sputum tests were negative for tuberculosis. Screen examination showed that both diaphragmatic movements were poor, and the film showed only advanced emphysema with no evidence of infiltration of lung parenchyma or even of chronic bronchitis; the bases were clear and had no abnormal striation suggestive of enlargement of the lower bronchi. 


\section{Commentary.}

Aids to diagnosis in this case are mainly negative. The history of increasing cough and sputum, loss of weight, night sweats, general malaise and increasing breathlessness would suggest the diagnosis of chronic phthisis, and the finding of a generalized bronchitis, even if it had been mainly basal, would not have ruled it out. On the other hand, the normal pulse and temperature, negative sputum tests, and lack of dullness and of signs of fibrosis or displacement of mediastinum and heart, which would have accompanied the poor general condition in such a long standing history, must militate against it.

Similar signs must have been found with foreign body in a bronchus, and while the loss of weight, negative sputum, normal pulse and temperature, increasing breathlessness even when walking on the flat, together with the normal position of the heart and mediastinum in the presence of a generalized bronchitis, would all favour a diagnosis of bronchial or true pulmonary carcinoma, the length of the history and the absence of any area of dead dullness and the normal $\mathrm{X}$-ray picture are against it.

By a process of exclusion the only possible diagnosis was that the patient was suffering from a " silent bronchiectasis." In such cases no added sounds are heard in the lungs, as the dilated bronchi and cavities are full of pus. Even in such cases there is usually marked dullness at one or both bases, but the gross emphysema in this case would mask such dullness, and it is well known that when bronchiectatic cavities are full of pus they do not give the usual dry, hard, metallic crepitations, heard in the first stage of inspiration. It was therefore decided to try postural drainage, and this produced about half a pint of sputum which was mainly pus, and which the patient described as having a nasty, sweetish taste and a stale earthy smell which he had not noted in his morning sputum. Lipiodol injection bore out the diagnosis, the film showing gross dilatations with early sacculations of the bronchi at both bases.

The patient was seen six months later. On routine postural drainage, rest, and tonics with creosote (minim one in capsule) three times a day, his general condition had improved enormously; his appetite was better and he had added seven pounds in weight, while the decreasing amount of sputum which he had noted in the last four months had lost its offensive taste and smell. Such an advanced bilateral dilatation of the bronchi is unsuitable for any active intervention and all that can be hoped for is that its progress may be kept under control by the general medical measures mentioned. With improving general condition he might now gain distinct benefit by slowly increased treatments in a creosote chamber, but bronchoscopic suction and lavage is not likely to benefit; fortunately he had not reached the stage of gross sacculation and cavity formation.

Case presented by R. R. TraIL, M.C., M.D., F.R.C.P. 